CYSTIC FIBROSIS

\title{
Controlled longitudinal study of bone mass accrual in children and adolescents with cystic fibrosis
}

\author{
H M Buntain, P J Schluter, S C Bell, R M Greer, J C H Wong, J Batch, P Lewindon, \\ C E Wainwright
}

See end of article for authors' affiliations

.....................

Correspondence to: Dr H Buntain, Department of Respiratory Medicine, Royal Children's Hospital, Herston, QLD 4029,

Australia; scottmel@

bigpond.net.au

Received 14 May 2005

Accepted

21 November 2005

Published Online First

29 December 2005

\begin{abstract}
Background: A study was undertaken to observe the gains in bone mass in children and adolescents with cystic fibrosis (CF) over 24 months and to examine the relationship between areal bone mineral density (aBMD) and associated clinical parameters including physical activity, nutrition, and 25-hydroxyvitamin D (25OHD).

Methods: Areal BMD of the total body (TB), lumbar spine (LS), and total femoral neck (FNt) were repeatedly measured in 85 subjects aged 5-18 years with CF and 100 age and sex matched controls over 2 years. At each visit anthropometric variables, nutritional parameters, pubertal status, disease severity, physical activity, dietary calcium, caloric intake, and serum 25OHD were assessed and related to aBMD. Results: After adjusting for age, sex, and height Z-score, gains in LS ABMD in children (5-10 years) and $\mathrm{TB}$ and FNt aBMD in adolescents (11-18 years) with CF were significantly less than in controls. Lean tissue mass was significantly associated with TB and LS aBMD gains in children and adolescents and explained a significant proportion of the aBMD deficit observed. Lung function parameters were significantly associated with $\mathrm{aBMD}$ gains in adolescents with $\mathrm{CF}$.

Conclusions: Inadequate bone mass accrual during childhood and adolescence contributes to the low bone mass observed in adults with CF. Accounting for the height discrepancy which is frequently observed in those with CF, in addition to age and sex, is important when assessing low bone mass in children and adolescents with CF. To optimise an individual's potential to acquire maximal bone mass, it is necessary to maximise nutritional status and limit the progression of chronic suppurative lung disease.
\end{abstract}

A survival improves for those with cystic fibrosis (CF), it is becoming increasingly important to understand the evolution of low bone mineral density (BMD) which has been extensively reported in adults with CF. ${ }^{1-5}$ The association between low BMD and increasing incidence of fracture has been well documented in the elderly of the general population. ${ }^{67}$ Higher fracture incidences, in particular vertebral fractures, have been reported in adolescent females $^{8}$ and adults with CF. ${ }^{3-11}$ The detrimental effects of a fracture in an individual with CF includes pain and the impedance of effective treatment directed towards the management of their suppurative lung disease.

Bone mass accrual is a continuous process from birth through childhood and adolescence. Rapid gains in bone mass occur during adolescence with approximately 26\% being acquired during the 2 years surrounding peak height velocity. ${ }^{12-14}$ Peak bone mass is reached in late adolescence and the achieved bone mass then acts as the bone bank for the remainder of life. In children and adolescents with CF, normal to low BMD has been reported in cross sectional studies $^{11}{ }^{15-25}$ and bone mass accrual has varied from normal to reduced in longitudinal studies. ${ }^{1}{ }^{41125}$ Many of these studies have involved small patient cohorts and have not accounted for the variations in growth parameters in children and adolescents with CF.

Bone mineral density is a function of bone mineral content (BMC) per unit volume of bone $\left(\mathrm{g} / \mathrm{cm}^{3}\right)$. The use of dual energy $x$ ray absorptiometry (DXA) is associated with low radiation exposure, hence its frequent use in children and young people, especially healthy individuals. However, DXA measures BMC and bone area and then calculates areal bone mineral density $(\mathrm{aBMD})$ as $\mathrm{BMC} /$ bone area $\left(\mathrm{g} / \mathrm{cm}^{2}\right)$. Areal $\mathrm{BMD}$ is a two dimensional measurement that does not correct for the depth or size of bone and is thus age and size dependent in growing individuals. ${ }^{26-28}$ The finding of reduced
aBMD in a "short" individual may be due either to small bones or less mineral content. Although the problem is widely appreciated, there is no consensus as to the most appropriate way to correct aBMD measurements for size. Several different approaches have been suggested. ${ }^{28-30}$ One approach is the use of multiple regression analysis where aBMD is simultaneously adjusted for age, height, and other relevant factors. This method is appropriate for research. A second option is a staged approach where height is adjusted for age distinguishing "short" bones, next bone area is adjusted for height distinguishing "narrow" bones, and BMC is adjusted for bone area distinguishing "thin" bones. This approach is easily applied to clinical situations as the clinician can compare a patient's aBMD and height measurements with centile curves as outlined by Molgaard.$^{28}$ The final option is to calculate volumetric bone density (bone mineral apparent density, BMAD) where BMC is adjusted for a calculated bone volume rather than bone area. ${ }^{29}$ This method attempts to adjust for a third dimension that DXA does not capture $^{262731}$ and is not applicable for total body measures of aBMD. ${ }^{29}$ Short stature is frequently observed in adolescents with CF and hence needs to be accounted for when assessing aBMD measurements.

Bone consists of cortical and trabecular (or cancellous) bone. Cortical bone composes $\sim 85 \%$ of the total body's bone and is controlled predominantly by systemic osteotrophic hormones including growth hormone, parathyroid hormone, and 1,25-dihydroxyvitamin $\mathrm{D}\left(1,25(\mathrm{OH})_{2} \mathrm{D}\right) .{ }^{29}{ }^{32}$ In contrast, the lumbar spine consists of $\sim 65 \%$ trabecular bone and

Abbreviations: $a B M D$, areal bone mineral density; $B M C$, bone mineral content; $\mathrm{CF}$, cystic fibrosis; $\mathrm{FEV}_{1}$, forced expiratory volume in 1 second; FNt, total femoral neck; FVC, forced vital capacity; LS, lumbar spine; LTM, lean tissue mass; 25OHD, 25-hydroxyvitamin $\mathrm{D} ; 1,25(\mathrm{OH})_{2} \mathrm{D}$, 1,25-dihydroxyvitamin $\mathrm{D}$; TB, total body 
$\sim 35 \%$ cortical bone. Trabecular bone is more sensitive to corticosteroids and inflammatory cytokines. ${ }^{29}{ }^{32} 33$ It is important to measure both total body and lumbar spine bone mass in children and young people with chronic disease in an attempt to understand the underlying pathological processes.

The pathophysiology and timing of evolution of low BMD in adults with CF is not understood. Physical activity and dietary calcium are well known to influence bone mass accrual in healthy individuals. ${ }^{12} 34{ }^{35}$ Young people with CF may be less physically active as a consequence of their pulmonary status. Other unique features which may impede bone mass accrual in those with CF include chronic inflammation, suboptimal nutritional status secondary to pancreatic insufficiency, corticosteroid usage, inadequate steroid hormone levels during puberty, ${ }^{36}$ and possibly the effect of the dysfunctional CF transmembrane conductance regulator (CFTR) on bone turnover.

The aims of this study were to observe whether bone mass accrual, as measure by total body and lumbar spine aBMD, in children and adolescents with CF were comparable to local age and sex matched healthy controls. The second aim was to correlate aBMD gains with the clinical factors known to influence bone mass accrual, with special emphasis on nutritional status, vitamin D status, and physical activity.

\section{METHODS}

All children and adolescents aged 5-18 years with CF who attended the Royal Children's Hospital, Brisbane (RCH) CF clinic were approached to partake in this longitudinal study. Patients were excluded if they had a primary bone disorder or were awaiting lung transplantation. The diagnosis of CF had been confirmed by an elevated sweat chloride test. For comparison, healthy controls were recruited by approaching 148 local schools to distribute an information leaflet about the study. Interested individuals then contacted the study coordinator. Exclusion criteria included chronic illness or a period of immobility of $\geqslant 2$ weeks during the preceding 12 months. Control subjects were screened by history and laboratory examination to exclude chronic illness. Limited published data on bone mass accrual in children and adolescents with CF adjusted for age, sex, and height prevented power calculations at the outset of the study. Withdrawal rates were anticipated to be greater among control subjects, so $15 \%$ more controls were recruited.

The results from the initial review have been reported in conjunction with additional adolescent and adults subjects who attended The Prince Charles Hospital (TPCH) CF clinic. ${ }^{17}$ Subjects attending TPCH did not participate in the longitudinal analysis. Study recruitment occurred between March 2000 and December 2001. Ethical approval was obtained from the RCH research and ethics committee. Written informed consent was obtained from the subjects' parents and additionally from subjects aged 10 years or older.

At each visit a series of investigations was performed as detailed below. Study visits were performed at 6 monthly intervals for CF subjects on five occasions and at 12 monthly intervals for controls on three occasions.

\section{Clinical parameters}

Weight was measured using a calibrated digital scale $(\mathrm{CH}-$ $150 \mathrm{kP}$, A\&D Mercury Pty Ltd) to the nearest $10 \mathrm{~g}$ and height with a wall mounted stadiometer (Magnimetre, Raven Equipment Ltd, UK) to the nearest $1 \mathrm{~mm}$ by $\mathrm{HB}$ or $\mathrm{BD}$ (laboratory technician). Height and weight were expressed as Z-scores (age and sex specific standard deviation scores) based on World Health Organisation normal data. ${ }^{37}$ Subjects with CF performed spirometric tests in the respiratory investigation unit in accordance with the American Thoracic Society standards. The best recorded forced expiratory volume in 1 second $\left(\mathrm{FEV}_{1}\right)$ and forced vital capacity (FVC) during the 12 months prior to and including the first study visit were recorded and expressed as a percentage of the predicted value. ${ }^{38}$ For subsequent visits the best $\mathrm{FEV}_{1}$ and FVC for the period between and including the study visit were recorded. If a subject with CF missed a study visit, clinical parameters from a clinic visit within a month of the study visit due date were recorded if available. Each participant self assessed their pubertal status according to the method of Tanner by comparing illustrations of pubertal development without parental influence. ${ }^{39}$ This method is reported to correlate well with the results of physical examination by a physician. ${ }^{40}$ Age at menarche was recorded for females by historical recall from the individual.

Using a standardised booklet, RCH subjects completed a 7 day food intake diary (as children can vary their diet substantially from day to day). Mean daily calcium and caloric intake were determined by a single dietician with the use of Foodworks Version 2.10.136 (Xyris Software (Australia) Pty Ltd). The daily caloric intake for control subjects was expressed as a percentage of the recommended daily caloric intake for Australian children. ${ }^{41}$ For CF subjects, $120 \%$ of the recommended daily caloric intake was used in accordance with standard recommendations for CF care. The validated "Physical activity questionnaire for older children (9-15 years)" was used. ${ }^{42}$ This is a self-administered 7 day recall questionnaire with nine items, each scored on a 5-point Likert scale and used to derive a total activity score.

\section{Bone densitometry measurements}

Areal BMD of the total body (TB), lumbar spine (L2-4) (LS) and total femoral neck (FNt), total body bone mineral content (TB BMC) and lean tissue mass (LTM) were measured by DXA with a Lunar DPX-L bone densitometer using paediatric software (Version 4.7e, Lunar Corp, Madison, WI, USA) and expressed as $\mathrm{g} / \mathrm{cm}^{2}$. Total body and LS aBMD were also expressed as Z-scores using the Lunar normative database which has received contributions from several Australian sites. ${ }^{27}$ The departmental short term precision for adults at each site was $1 \%$ for the LS and TB measurements and $1.5 \%$ for FNt. The LTM precision, as determined in a phantom, was less than $1 \%$.

\section{Laboratory measurements}

Random non-fasting blood samples were collected at the initial study visit only for controls and at each study visit for CF subjects. 25-hydroxyvitamin D (25OHD; nmol/l) and 1,25hydroxyvitamin $\mathrm{D}\left(\mathrm{l}, 25(\mathrm{OH})_{2} \mathrm{D}\right.$, pmol/l) were measured using DiaSorin RIA Double Antibody assay (DiaSorin, Stillwater, Minnesota, USA) by Queensland Health Pathology Services (QHPS) (Royal Brisbane Hospital, Herston, Australia).

\section{Historical data}

Pancreatic sufficiency status, the presence of CF related liver disease (defined as the presence of multinodular cirrhosis and portal hypertension), and the presence of CF related diabetes mellitus were collected by historical recall and chart review. The following information was collected for the 12 months prior to the study and for each study period: (1) inhaled and oral corticosteroid usage expressed as the mean daily dose in $\mu \mathrm{g} / \mathrm{kg} /$ day and $\mathrm{mg} / \mathrm{kg} /$ day, respectively; (2) number of chest exacerbations (defined as an increase in cough or sputum production sufficient to warrant change in antibiotic therapy); (3) number of admissions; and (4) total number of days in hospital. Fluticasone has greater potency, greater glucocorticoid receptor binding affinity, and a longer half life than beclomethasone and budesonide, ${ }^{43}$ so the 


\begin{tabular}{|c|c|c|c|c|c|}
\hline & \multicolumn{2}{|l|}{$\mathrm{CF}$} & \multicolumn{2}{|c|}{ Control } & \multirow[b]{2}{*}{ p value } \\
\hline & $\mathrm{N}$ & Mean (SD) & $\mathrm{N}$ & Mean (SD) & \\
\hline \multicolumn{6}{|l|}{ Children } \\
\hline$F / M$ & 40 & $21 / 19$ & 32 & $18 / 14$ & 0.81 \\
\hline Age (years) & 40 & $8.5(1.8)$ & 32 & $8.5(1.5)$ & 0.89 \\
\hline Weight Z-score & 40 & $0.02(0.97)$ & 32 & $0.28(1.09)$ & 0.28 \\
\hline Height Z-score & 40 & $-0.22(0.95)$ & 32 & $0.21(1.06)$ & 0.10 \\
\hline Total body BMD Z-score & 40 & $0.23(0.74)$ & 32 & $0.26(0.70)$ & 0.87 \\
\hline Lumbar spine BMD Z-score & 40 & $-0.28(0.95)$ & 32 & $-0.31(0.99)$ & 0.88 \\
\hline $\mathrm{FEV}_{1}(\%)$ median (range) & 39 & $84(46-116)$ & & & \\
\hline FVC (\%) median (range) & 39 & $93(53-122)$ & & & \\
\hline \multicolumn{6}{|l|}{ Adolescents } \\
\hline$N(F / M)$ & 45 & $18 / 27$ & 68 & $36 / 32$ & 0.19 \\
\hline Age (years) & 45 & $14.1(2.2)$ & 68 & $13.9(1.9)$ & 0.47 \\
\hline Weight Z-score & 45 & $-0.75(0.91)$ & 68 & $0.26(0.82)$ & $<0.001$ \\
\hline Height Z-score & 45 & $-0.87(0.84)$ & 68 & $0.59(0.97)$ & $<0.001$ \\
\hline Total body BMD Z-score & 45 & $-1.29(1.48)$ & 68 & $0.37(0.93)$ & $<0.001$ \\
\hline Lumbar spine BMD Z-score & 45 & $-0.53(1.22)$ & 68 & $-0.18(1.24)$ & $<0.001$ \\
\hline $\mathrm{FEV}_{1}(\%)$ median (range) & 45 & $86(23-124)$ & & & \\
\hline FVC (\%) median (range) & 45 & $90(36-123)$ & & & \\
\hline
\end{tabular}

Values are mean (SD) unless otherwise stated.

$\mathrm{BMD}$, bone mineral density; FEV 1 , forced expiratory volume in 1 second.

fluticasone dose was doubled to be dose equivalent to beclomethasone. No adjustment was made for budesonide.

\section{Statistical analysis}

The sample was divided into two age groups: (a) children (510 years including self-assessed Tanner stage $\leqslant 2$ at the study commencement) and (b) adolescents ( $11-18$ years) to account for the pubertal growth spurt. ${ }^{13} 1444$ Student's $t$ test, Fisher's exact test, and Wilcoxon rank sign test were used to compare groups where appropriate. Generalised estimating equations (GEE) assuming normal responses were used to estimate and test predictor variables over time. This method allows all available data to be used in the analysis, including data from those who withdrew from the study. Relationships between successive observations were modelled using an exchangeable correlation matrix. ${ }^{45}$ Robust variance estimate techniques were used to calculate standard errors and confidence intervals. ${ }^{45}$ Models relating aBMD between groups ( control $=0, \mathrm{CF}=1$ ) were generated for each age group. Age for children and adolescents were categorised into quartiles. These models were initially adjusted for age quartile, sex, and age $\times$ sex interactions to correct for the differences in age and sex between study participants. Models for aBMD were then additionally adjusted for height Z-score. The regression $\beta$ estimates for the predictor variables "group", "time", and "time $\times \mathrm{CF}$ " have been reported and give the direction and size of the difference between CF and control groups over time. To determine the adequacy of the sample size, the detectable difference between CF and control groups was calculated from the available study numbers, the correlation observed between repeated observations, and the standard deviation of the outcome variables using the method outlined by Twisk ${ }^{45}$ applying a significance level of $5 \%$ and power of $80 \%$. Subject withdrawal and non-attendance were also examined by GEE models using a binomial outcome $(0=$ value present, $\mathrm{l}=$ value missing $)$ and a logit link function to assess for differences between the CF and control groups. ${ }^{46}$ Baseline clinical parameters and aBMD adjusted for age, sex, and height Z-score were compared between those who completed and those who withdrew from the study. Pubertal development for each sex was analysed using GEE models adjusted for age. Seasonal variation (month of blood collection: 1 = September to April, 2 = May to August) was included in the GEE models for 25OHD. To determine the contribution of clinical variables on bone mass accrual, each covariate was added to the TB and LS aBMD GEE models adjusted for age, sex, and height Z-score. Some of the variables are only applicable to those with $\mathrm{CF}$, so further GEE models were generated only including CF subjects. Using these models, all variables significantly associated with aBMD were combined to determine the clinical variables that had the greatest influence on aBMD accrual in those with CF. Data were analysed using Stata Version 8. Significance was set at a $\mathrm{p}$ value of $<0.05$.

\section{RESULTS}

One hundred and fifty seven children and adolescents with CF were identified as being eligible, of whom 85 agreed to participate. The subject characteristics are outlined in tables 1 and 2 . The mean height Z-score at study commencement was significantly lower in adolescents who participated in the study than in those who, although eligible, elected not to participate. The mean height Z-score was not significantly different from the mean values for young people with CF in Australia (data not shown). ${ }^{47}$ There were no differences in the mean weight $\mathrm{Z}$-score and $\mathrm{FEV}_{1}$ in $\mathrm{CF}$ participants compared with non-participants or the mean values for young people with CF in Australia (data not shown). ${ }^{47}$

Of the 85 subjects with CF and 100 controls who enrolled in the study, 65 and 83 individuals respectively completed the study (fig 1). The occurrence of missing values between CF and control children were similar $(p=0.50)$, however missing values were more likely in adolescents with CF than controls $(p=0.02)$. Areal aBMD (adjusted for age, sex, and

Table 2 Cystic fibrosis specific features at the commencement and completion of the study

\begin{tabular}{lll}
\hline & $\begin{array}{l}\text { Initial review } \\
\mathbf{N}(\%)\end{array}$ & $\begin{array}{l}\text { Study completion } \\
\mathbf{N}(\%)\end{array}$ \\
\hline CF related diabetes mellitus & $3(4)$ & $5(6)$ \\
Insulin dependent diabetes mellitus & 1 & 1 \\
CF related liver disease & $15(18)$ & $17(20)$ \\
Pancreatic sufficient & $5(6)$ & $4(5)$ \\
\hline
\end{tabular}




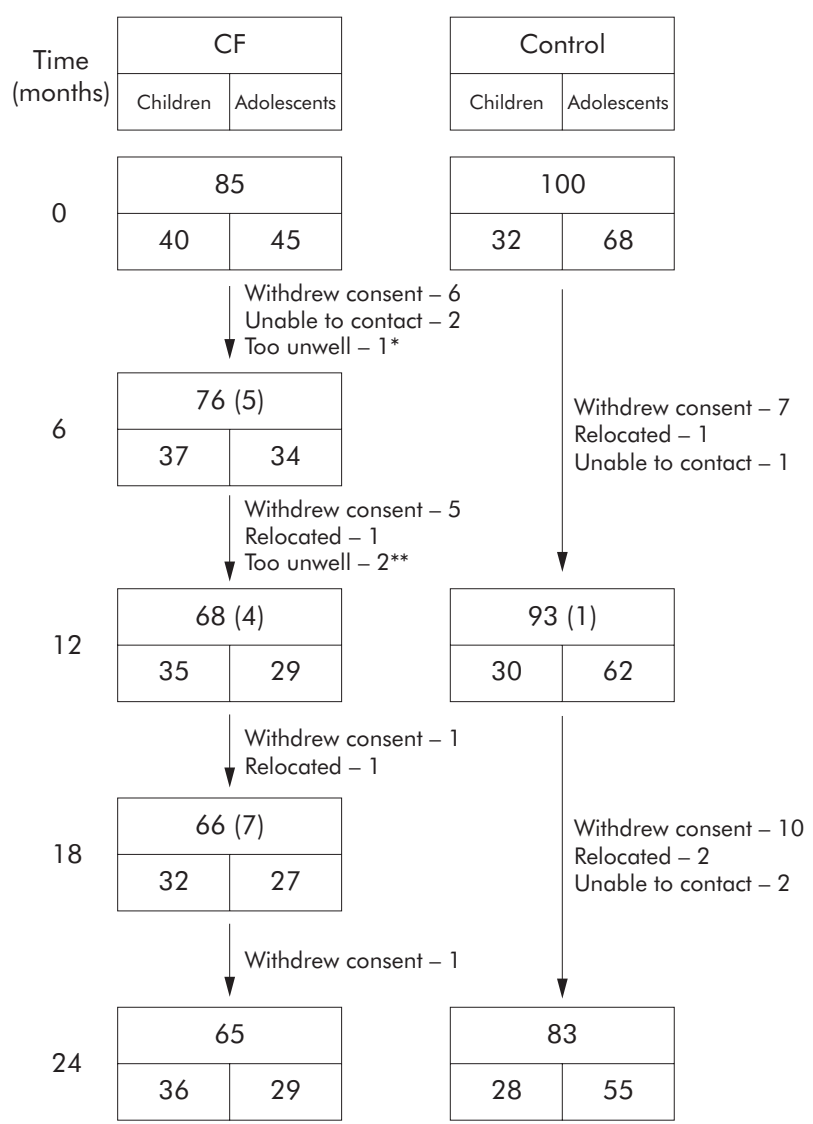

*Adolescent male died 11 months into the study

**Adolescent male identical twins. Died 12.5 and 16.5 months into the study

Figure 1 Study participation path showing the number of individuals in the study at each visit (number who missed the review through remained in the study) and the number in each age group who completed each review.

height Z-score), height, and FVC at study commencement were similar between those who completed and those who withdrew from the study in both children and adolescents with CF. However, $\mathrm{FEV}_{1}$ was lower in adolescents who withdrew from the study (median (IQR) 92\% (27-124) in those who completed the study and $78 \%(23-120)$ in those who withdrew, $\mathrm{p}=0.03$ ).

\section{Longitudinal change in areal bone mineral density}

With the available subject numbers, the study was powered to detect a difference in aBMD gain between CF and control groups for children and adolescents of $0.03 \mathrm{~g} / \mathrm{cm}^{2}$ for TB aBMD and $0.05 \mathrm{~g} / \mathrm{cm}^{2}$ for LS aBMD. The mean (SD) gain in aBMD in control children for TB was $0.07(0.03) \mathrm{g} / \mathrm{cm}^{2}$ and for LS was $0.09(0.04) \mathrm{g} / \mathrm{cm}^{2}$, while in control adolescents the mean gain for TB was $0.10(0.05) \mathrm{g} / \mathrm{cm}^{2}$ and for LS was 0.13 $(0.08) \mathrm{g} / \mathrm{cm}^{2}$. The aBMD longitudinal changes in children and adolescents are shown in tables 3 and 4 and illustrated in fig 2 .

At study commencement there were no differences in aBMD (adjusted for age and sex) at any of the three measured sites between CF and control children. Over time, aBMD increased significantly in both study groups at all measured sites as expected with growth. However, the aBMD gain at the LS was significantly less in children with CF relative to controls (table 3 ). These results did not change when adjusted for height. Adolescents with CF had lower aBMD (adjusted for age and sex) than controls at all three measured sites at study commencement. Areal BMD increased in both study groups over time and there was no difference in the rate of aBMD accrual between CF and controls groups (table 4 and fig 2). However, when aBMD was adjusted for height, TB and FNt aBMD gains were lower in adolescents with CF than in controls.

Similar results were observed for TB BMC to those observed for TB aBMD when analysed using the same approach (data not shown).

\section{Longitudinal change in clinical parameters}

The mean gain in weight over 24 months was less in children with CF than in control subjects, although height and LTM gains were similar between the two groups (table 5). In adolescents the gains in weight and LTM were similar in the $\mathrm{CF}$ and control groups while the gain in height was nonsignificantly greater in CF subjects than in controls. Lung function parameters, $\mathrm{FEV}_{1}$, and FVC declined significantly in adolescents with CF but not in children (table 5). There were similar rates of pubertal development in $\mathrm{CF}$ and control girls during the study (breast development: time $\times \mathrm{CF}, \mathrm{p}=0.28$ ). Despite the fact that boys with CF had delayed pubertal staging relative to controls at study commencement, there

Table 3 Longitudinal comparison of total body, lumbar spine, and total femoral neck areal bone mineral density in children (5-10 years)

\begin{tabular}{|c|c|c|c|c|c|}
\hline \multirow[b]{2}{*}{ Site measured } & \multirow[b]{2}{*}{ Parameter } & \multicolumn{2}{|l|}{ Adjusted for age and sex* } & \multicolumn{2}{|l|}{ Additionally adjusted for height† } \\
\hline & & $\begin{array}{l}\text { B estimate } \\
(95 \% \mathrm{Cl})\end{array}$ & $p$ value & $\begin{array}{l}\text { B estimate } \\
(95 \% \mathrm{Cl})\end{array}$ & $p$ value \\
\hline Total body & $\begin{array}{l}\text { Group } \\
\text { Time } \\
\text { Time } \times \text { CF }\end{array}$ & $\begin{array}{l}-0.007(-0.033 \text { to } 0.019) \\
0.003(-0.033 \text { to } 0.019) \\
-0.001(-0.001 \text { to }-0.000)\end{array}$ & $\begin{array}{l}0.59 \\
<0.001 \\
0.05\end{array}$ & $\begin{array}{l}-0.005(-0.031 \text { to } 0.020) \\
0.003(0.003 \text { to } 0.003) \\
-0.001(-0.001 \text { to } 0.000)\end{array}$ & $\begin{array}{l}0.68 \\
<0.001 \\
0.07\end{array}$ \\
\hline Lumbar spine & $\begin{array}{l}\text { Group } \\
\text { Time } \\
\text { Time } \times \text { CF }\end{array}$ & $\begin{array}{l}0.003(-0.043 \text { to } 0.050) \\
0.004(0.003 \text { to } 0.005) \\
-0.001(-0.002 \text { to }-0.000)\end{array}$ & $\begin{array}{l}0.89 \\
<0.001 \\
0.02\end{array}$ & $\begin{array}{l}0.007(-0.038 \text { to } 0.053) \\
0.004(0.003 \text { to } 0.005) \\
-0.001(-0.002 \text { to }-0.000)\end{array}$ & $\begin{array}{l}0.76 \\
<0.001 \\
0.04\end{array}$ \\
\hline Femoral neck (total) & $\begin{array}{l}\text { Group } \\
\text { Time } \\
\text { Time } \times \text { CF }\end{array}$ & $\begin{array}{l}-0.004(-0.061 \text { to } 0.052) \\
0.004(0.003 \text { to } 0.006) \\
-0.002(-0.004 \text { to } 0.000)\end{array}$ & $\begin{array}{l}0.88 \\
<0.001 \\
0.08\end{array}$ & $\begin{array}{l}0.013(-0.038 \text { to } 0.064) \\
0.004(0.003 \text { to } 0.006) \\
-0.002(-0.004 \text { to } 0.001)\end{array}$ & $\begin{array}{l}0.63 \\
<0.001 \\
0.15\end{array}$ \\
\hline
\end{tabular}

*Adjusted for age quartile, sex, and age quartile $\times$ sex interaction.

†Adjusted for age quartile, sex, age quartile $\times$ sex interaction, and height Z-score.

Areal bone mineral density adjusted for age and sex and then additionally for height. The $\beta$ estimate with associated $95 \%$ confidence intervals $(95 \% \mathrm{CI})$ for group (control = $0, C F=1$ ), time, and time $\times C F$ are reported. "Group" represents the difference (CF - control) at study commencement, "time" represents the change in $\mathrm{aBMD}$ over time for the whole group, and "time $\times \mathrm{CF}^{\prime \prime}$ represents the difference in change for aBMD between the CF and control groups over time. 
was no change in the difference over time (genital development: time $\times \mathrm{CF}, \mathrm{p}=0.28)$. At study completion, $17(55 \%)$ girls with CF and $32(68 \%)$ controls aged 10 years and older had reached menarche $(p=0.34)$. The median age for menarche was similar for the two groups (CF: 13.0 years (range 10-15), controls: 13.0 years (range 11-16.3); $\mathrm{p}=0.90)$. None of the female participants were taking the oral contraceptive pill during the study.

There was significant seasonal variation associated with 25OHD for both children $(p=0.001)$ and adolescent $(p<0.001)$ CF subjects. Accounting for seasonal variation, the mean 25OHD in children with CF declined significantly from 64.0 (15.5) nmol/l to 55.6 (13.1) nmol/l, and in adolescents with CF from 61.3 (15.2) nmol/l to 54.7 (17.8) $\mathrm{nmol} / \mathrm{l}$ (table 5).

Completion of the diet diaries at each study visit varied from $42 \%$ to $73 \%$. At study commencement, calcium intake in both children and adolescents with CF was significantly greater than in controls and remained as such throughout the study (table 5). Completion of the activity questionnaire at each visit varied from $40 \%$ to $87 \%$. The mean activity questionnaire score was significantly higher in children with CF than in controls at study commencement and did not change significantly during the study. In adolescents, similar activity scores were observed between CF and control subjects at study commencement although they declined significantly in the adolescent group as a whole. There was no significant difference in the rate of decline between CF and control groups.

\section{Corticosteroid exposure}

Inhaled corticosteroids were used more frequently in those with CF. In children with CF, inhaled corticosteroid usage
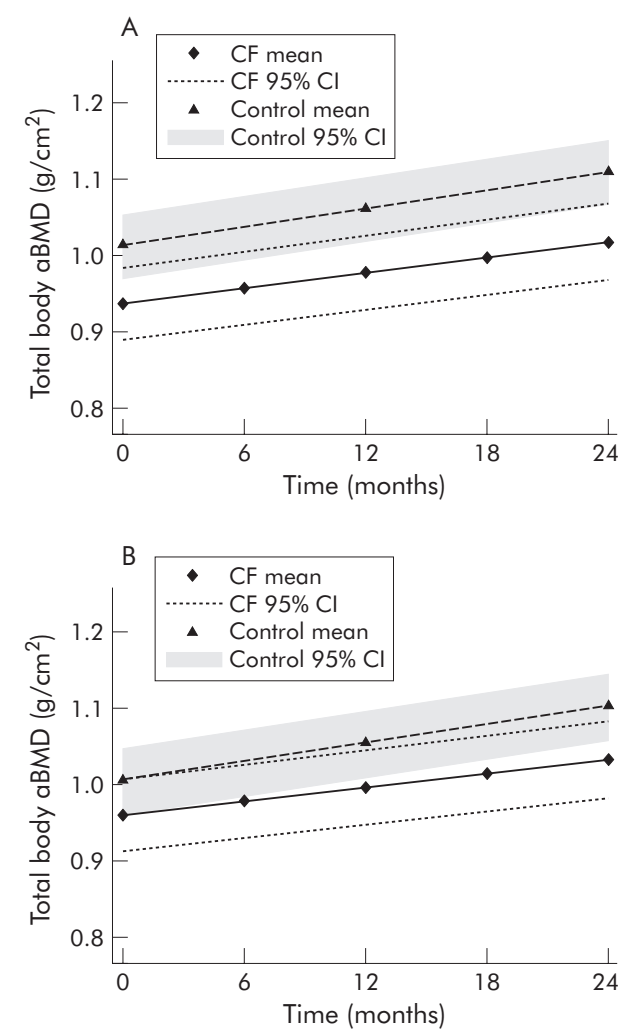

Figure 2 Longitudinal changes in total body areal bone mineral density in adolescents. (A) Adjusted mean with associated $95 \%$ confidence intervals for age and sex. (B) Adjusted mean with associated $95 \%$ confidence intervals for age, sex and height Z-score. increased from $78 \%$ at study commencement to peak at $97 \%$ during the fourth visit, while in adolescents the reverse trend was observed with $94 \%$ using inhaled corticosteroids at the second visit and declining to $76 \%$ at the last visit. Control subject usage of corticosteroids ranged from $4 \%$ to $16 \%$. There was a dosage reduction for both children and adolescents with CF over the duration of the study, with the median (IQR) dose decreasing from $46.2(28.2-69.5) \mu \mathrm{g} / \mathrm{kg} /$ day at study commencement to $22.9 \quad(13.7-57.5) \mu \mathrm{g} / \mathrm{kg} / \mathrm{day}$ at completion in children and from $30.7(21.8-49.4) \mu \mathrm{g} / \mathrm{kg} / \mathrm{day}$ to $22.5(18.4-30.5) \mu \mathrm{g} / \mathrm{kg} /$ day in adolescents.

Fewer control participants took oral corticosteroids during the study with usage ranging from 0 to $6 \%$ in children and adolescents at each visit. One control used oral corticosteroids for a period of 4 weeks for the management of Bell's palsy. In those with CF, the percentage taking oral corticosteroids at each visit ranged from $22 \%$ to $35 \%$ in children and from $11 \%$ to $28 \%$ in adolescents. Only two individuals with CF took oral corticosteroids for more than $50 \%$ of the study period.

\section{Fracture incidence}

During the 2 year period before commencement of the study 10 fractures occurred in 10 subjects with CF and 15 in 10 control individuals $(\mathrm{p}=0.81)$. During the study period the fracture frequencies were similar in CF and control subjects with seven and eight fractures occurring in CF and control subjects, respectively $(\mathrm{p}=1.00)$.

\section{Clinical associations with areal bone mineral density: longitudinal analysis}

Lean tissue mass was a consistent significant covariate in all of the aBMD models (including $\mathrm{CF}$ and control groups adjusted for age, sex, and height Z-score) for both children and adolescent groups (children: TB: $p=0.02, \mathrm{LS}$ : $\mathrm{p}<0.001$; adolescents: TB: $\mathrm{p}<0.001$, LS: $\mathrm{p}<0.001$ ). With the inclusion of LTM in the aBMD models, aBMD accrual over time was no longer significantly different between $\mathrm{CF}$ and control groups ("time $\times \mathrm{CF}^{\prime}$ in children: TB $\mathrm{p}=0.10$, LS $\mathrm{p}=0.08$; adolescents: TB $p=0.05$, LS $p=0.14)$. The only other significant covariate was the percentage of the recommended daily caloric intake for TB aBMD in children $(p=0.01)$. No significant associations were found between adjusted TB or LS aBMD and activity questionnaire score or daily calcium intake in either age group when CF and control subjects were examined together.

The following associations were only examined in CF subjects. In children, FVC was significantly associated with adjusted TB aBMD $(\mathrm{p}=0.005)$ while $\mathrm{FEV}_{1}$ and $\mathrm{FVC}$ were both significantly associated with adjusted aBMD in adolescents $\left(\mathrm{FEV}_{1}\right.$ : TB: $\mathrm{p}<0.001$, LS: $\mathrm{p}<0.001$; FVC: TB: $\mathrm{p}<0.001$, LS: $p<0.001)$. No associations were found between adjusted aBMD and inhaled corticosteroids, oral corticosteroids, the number of exacerbations, the number of admissions, or the number of days in hospital.

Including all significant covariates identified to the adjusted aBMD model, final models were generated in CF subjects only. In children only FVC remained a significant covariate for adjusted TB aBMD and LTM for adjusted LS $\mathrm{aBMD}$. Lung function parameters, $\mathrm{FEV}_{1}$ and FVC were highly correlated so they were explored independently in adolescents with CF. Lean tissue mass and $\mathrm{FEV}_{1}$ remained significant covariates in the adjusted TB and LS aBMD models.

\section{DISCUSSION}

This large controlled longitudinal study of aBMD accrual in children and adolescents with CF has found suboptimal gains in LS aBMD in children with CF and suboptimal gains in TB and FNt aBMD in adolescents with CF compared with a local 
Table 4 Longitudinal comparison of total body, lumbar spine, and total femoral neck areal bone mineral density in adolescents (11-18 years)

\begin{tabular}{|c|c|c|c|c|c|}
\hline \multirow[b]{2}{*}{ Site measured } & \multirow[b]{2}{*}{ Parameter } & \multicolumn{2}{|l|}{ Adjusted for age and sex* } & \multicolumn{2}{|l|}{ Additionally adjusted for heightt } \\
\hline & & $\begin{array}{l}\text { B estimate } \\
\text { (95\% CI) }\end{array}$ & $p$ value & $\begin{array}{l}\text { B estimate } \\
\text { (95\% CI) }\end{array}$ & $\mathrm{p}$ value \\
\hline Total body & $\begin{array}{l}\text { Group } \\
\text { Time } \\
\text { Time } \times \text { CF }\end{array}$ & $\begin{array}{l}-0.076(-0.107 \text { to }-0.046) \\
0.004(0.004 \text { to } 0.005) \\
-0.001(-0.002 \text { to } 0.000)\end{array}$ & $\begin{array}{l}<0.001 \\
<0.001 \\
0.17\end{array}$ & $\begin{array}{l}-0.046(-0.079 \text { to }-0.013) \\
0.004(0.004 \text { to } 0.005) \\
-0.001(-0.002 \text { to }-0.000)\end{array}$ & $\begin{array}{l}0.006 \\
<0.001 \\
0.03\end{array}$ \\
\hline Lumbar spine & $\begin{array}{l}\text { Group } \\
\text { Time } \\
\text { Time } \times \text { CF }\end{array}$ & $\begin{array}{l}-0.103(-0.149 \text { to }-0.058) \\
0.006(0.005 \text { to } 0.006) \\
-0.000(-0.002 \text { to } 0.001)\end{array}$ & $\begin{array}{l}<0.001 \\
<0.001 \\
0.54\end{array}$ & $\begin{array}{l}-0.041(-0.090 \text { to } 0.008) \\
0.005(0.005 \text { to } 0.006) \\
-0.001(-0.003 \text { to } 0.000)\end{array}$ & $\begin{array}{l}0.10 \\
<0.001 \\
0.07\end{array}$ \\
\hline Femoral neck (total) & $\begin{array}{l}\text { Group } \\
\text { Time } \\
\text { Time } \times \text { CF }\end{array}$ & $\begin{array}{l}-0.124(-0.176 \text { to }-0.072) \\
0.004(0.003 \text { to } 0.005) \\
-0.001(-0.003 \text { to } 0.001)\end{array}$ & $\begin{array}{l}<0.001 \\
<0.001 \\
0.20\end{array}$ & $\begin{array}{l}-0.066(-0.125 \text { to }-0.008) \\
0.004(0.003 \text { to } 0.005) \\
-0.002(0.003 \text { to } 0.005)\end{array}$ & $\begin{array}{l}0.03 \\
<0.001 \\
0.04\end{array}$ \\
\hline
\end{tabular}

*Adjusted for age quartile, sex, and age quartile $\times$ sex interaction.

†Adjusted for age quartile, sex, age quartile $\times$ sex interaction, and height Z-score.

Areal bone mineral density adjusted for age and sex and then additionally for height. The $\beta$ estimate with associated $95 \%$ confidence intervals ( $95 \% \mathrm{CI})$ for group (control = $0, C F=1$ ), time, and time $\times C F$ are reported. "Group" represents the difference (CF - control) at study commencement, "time" represents the change in $\mathrm{aBMD}$ over time for the whole group, and "time $\times \mathrm{CF}^{\prime}$ represents the difference in change for aBMD between the CF and control groups over time.

healthy control cohort after accounting for height discrepancies. These findings support the conclusion that the deficit in aBMD observed in adults with CF is in part due to inadequate bone mass accrual during childhood and adolescence.

To date, three longitudinal studies have reported aBMD in children and adolescents with CF. Bhudhikanok et al ${ }^{1}$ reported a significant reduction in TB aBMD Z-score for both females and males and LS aBMD Z-score for females over a mean period of 17 months compared with local controls. The mean weight and height gains were below normal which may explain some of the findings. More recently, Gronowitz et $a^{25}$ and Ujhelyi et $a l^{11}$ reported no significant change in LS or FN aBMD Z-scores over a 2 year study period despite both LS and FN aBMD Z-scores being significantly lower at study commencement. Neither of these studies used local healthy controls for comparison for the duration of the study.

Previous longitudinal studies ${ }^{11}{ }^{25}$ have not addressed the fundamental relationship between aBMD and height. In this study aBMD accrual adjusted for age and sex (equivalent to Z-scores) at all three measured sites in adolescents with CF were similar to controls, despite being significantly lower at study commencement; this is consistent with findings of previous studies. ${ }^{11}{ }^{25}$ With the addition of height to the aBMD models, TB and FNt aBMD accrual were significantly less in adolescents with CF than in controls, and LS aBMD also tended to be lower. These results highlight the importance of

Table 5 Longitudinal comparison of clinical measures in CF and control groups divided into two age groups: children (5-10 years) and adolescents (11-18 years)

\begin{tabular}{|c|c|c|c|c|c|c|}
\hline & \multicolumn{2}{|l|}{ Group } & \multicolumn{2}{|l|}{ Time } & \multicolumn{2}{|l|}{ Time $\times$ CF } \\
\hline & $\beta$ estimate $(95 \% \mathrm{Cl})$ & $p$ value & $\beta$ estimate $(95 \% \mathrm{Cl})$ & p value & $\beta$ estimate $(95 \% \mathrm{Cl})$ & $\mathrm{p}$ value \\
\hline \multicolumn{7}{|l|}{ Children } \\
\hline Weight $(\mathrm{kg})^{*} \dagger$ & $-1.01(-3.82$ to 1.81$)$ & 0.48 & $0.35(0.29$ to 0.40$)$ & $<0.001$ & $-0.09(-0.17$ to -0.01$)$ & 0.03 \\
\hline Height $(\mathrm{cm})^{*}$ & $-1.13(-4.06$ to 1.79$)$ & 0.45 & $0.51(0.46$ to 0.56$)$ & $<0.001$ & $-0.05(-0.12$ to 0.02$)$ & 0.14 \\
\hline Lean tissue mass $(\mathrm{kg})^{*}$ & $0.19(-0.92$ to 1.30$)$ & 0.73 & $0.20(0.17$ to 0.24$)$ & $<0.001$ & $-0.04(-0.09$ to 0.01$)$ & 0.13 \\
\hline Calcium intake (g/day) & $591.8(232.0$ to 951.6$)$ & 0.001 & $7.61(2.8$ to 12.5$)$ & 0.002 & $-2.3(-24.8$ to 19.6$)$ & 0.82 \\
\hline Recommended caloric intake (\%) & $22.06(-0.94$ to 45.07$)$ & 0.06 & $0.32(-0.25$ to 0.88$)$ & 0.27 & $-1.01(-2.37$ to 0.36$)$ & 0.15 \\
\hline Activity questionnaire & $0.45(0.12$ to 0.79$)$ & 0.008 & $0.01(-0.00$ to 0.02$)$ & 0.11 & $-0.02(-0.03$ to 0.00$)$ & 0.06 \\
\hline $\mathrm{FEV}_{1}$ (\% predicted) & & & $-0.20(-0.12$ to 0.01$)$ & 0.07 & & \\
\hline FVC (\% predicted) & & & $-0.16(-0.36$ to 0.03$)$ & 0.10 & & \\
\hline $25 \mathrm{OHD}(\mathrm{nmol} / \mathrm{l}) \ddagger$ & & & $-0.36(-0.52$ to -0.20$)$ & $<0.001$ & & \\
\hline $1,25(\mathrm{OH})_{2} \mathrm{D}(\mathrm{pmol} / \mathrm{l})$ & & & $-0.32(-0.93$ to 0.29$)$ & 0.30 & & \\
\hline \multicolumn{7}{|l|}{ Adolescents } \\
\hline Weight $(\mathrm{kg})^{*}$ & $-8.33(-11.6$ to -5.11$)$ & $<0.001$ & $0.35(0.28$ to 0.42$)$ & $<0.001$ & $-0.06(-0.16$ to 0.03$)$ & 0.17 \\
\hline Height $(\mathrm{cm})^{*}$ & $-9.58(-12.10$ to -7.06$)$ & $<0.001$ & $0.32(0.25$ to 0.39$)$ & $<0.001$ & $0.07(-0.04$ to 0.17$)$ & 0.20 \\
\hline Lean tissue mass $(\mathrm{kg})^{*}$ & $-6.01(-8.09$ to -3.93$)$ & $<0.001$ & $0.22(0.17$ to 0.27$)$ & $<0.001$ & $0.00(-0.07$ to 0.08$)$ & 0.91 \\
\hline Calcium intake (g/day) & $362.0(150.0$ to 574.1$)$ & $<0.001$ & $4.0(-0.5$ to 8.5$)$ & 0.08 & $-2.2(-11.7$ to 7.4$)$ & 0.66 \\
\hline Recommended caloric intake (\%) & $15.68(0.91$ to 30.45$)$ & 0.04 & $-0.44(-0.78$ to -0.10$)$ & 0.01 & $-0.77(-1.47$ to -0.07$)$ & 0.03 \\
\hline Activity questionnaire§ & $0.19(-0.09$ to 0.47$)$ & 0.18 & $-0.01(-0.02$ to -0.01$)$ & $<0.001$ & $-0.01(-0.02$ to 0.01$)$ & 0.41 \\
\hline $\mathrm{FEV}_{1}(\%$ predicted $)$ & & & $-0.36(-0.58$ to -0.14$)$ & 0.002 & & \\
\hline FVC (\% predicted) & & & $-0.25(-0.45$ to -0.05$)$ & 0.01 & & \\
\hline $250 \mathrm{OHD}(\mathrm{nmol} / \mathrm{l}) \ddagger$ & & & $-0.36(-0.64$ to 0.08$)$ & 0.06 & & \\
\hline $1,25(\mathrm{OH})_{2} \mathrm{D}(\mathrm{pmol} / \mathrm{l})$ & & & $-0.13(-0.67$ to 0.42$)$ & 0.65 & & \\
\hline
\end{tabular}

$\mathrm{FEV}_{1}$, forced expiratory volume in 1 second; FVC, forced vital capacity; $25 \mathrm{OHD}, 25$-hydroxyvitamin $\mathrm{D} ; 1,25(\mathrm{OH})_{2} \mathrm{D}, 1,25$ hydroxyvitamin $\mathrm{D}$.

*Variables adjusted for age, sex, and age $x$ sex interactions.

†Data required log transformation to obtain a normal distribution (untransformed results reported).

$\$ 25$ hydroxyvitamin $D$ adjusted for significant seasonal variation

$\S$ Activity questionnaire score adjusted for age due to significant interaction.

Group represents the difference (CF - control) at study commencement, time represents the change in aBMD over time for the whole group, and "time $\times$ CF" represents the difference between the CF and control groups in the changes in aBMD over time. There are no group comparisons available for lung function parameters or serum measures of vitamin D. 
adjusting for height when assessing aBMD measures in young people with $\mathrm{CF}$, particularly in the presence of short stature.

The mean aBMD gains in the control cohort were comparable to those reported in other healthy populations. ${ }^{13}$ With the available participants, the study was powered to detect a difference in aBMD gain over the study period between CF and control groups of $43 \%$ for TB and $56 \%$ for LS aBMD in children and $30 \%$ for TB and $38 \%$ for LS aBMD in adolescents. These detectable differences between CF and control groups are relatively large and clinically applicable differences in bone mass accrual between CF and control groups. These results should be taken into account for future study designs.

It is essential that the underlying pathology and pattern of evolution of low bone mass in individuals with CF should be determined to enable effective management strategies to be implemented. This task is difficult as many factors interplay with bone mass accrual ranging from inherited factors (including a possible role of dysfunctional CFTR) to the effects of chronic inflammation, malabsorption, and chronic disease impeding an active lifestyle. Access to bone histopathological evidence is limited, so much of our understanding to date has relied upon observational studies of $\mathrm{BMD}$. The findings of this study suggest that the underlying pathological process has a greater impairment on trabecular bone mass accrual initially as indicated by reduced LS aBMD gains in children, although it is more generalised during the growth phase experienced during adolescence as indicated by reduced gains in TB and FNt aBMD in adolescents with CF.

Lung function, a marker of disease severity, and LTM were the only consistent covariates associated with aBMD gains in this study, in agreement with others. ${ }^{1425}$ Body muscle delivers the largest load and strain to bone-influencing bone size, geometry and density-and LTM, a measure of muscle mass, is an independent predictor of bone mass in healthy subjects. ${ }^{48} 49$ Several studies have shown that aBMD and BMC in subjects with CF are similar to age, sex, height and LTM matched controls, despite a significant difference when compared with age and sex matched normative data. ${ }^{18} 1924$ In this study the lack of difference in aBMD gains between control and CF groups following inclusion of LTM implies that reduced muscle mass, in addition to short stature, explains a considerable proportion of the reduced bone mass accrual in children and adolescents with CF. Maximising nutritional status and body muscle mass in conjunction with limiting disease progression are important for optimising bone mass accrual.

A finding unique to this study is the clear documentation of normal pubertal progression in those with CF as indicated by clinical pubertal staging and age of menarche, which is different from previous studies. ${ }^{11}{ }^{11}{ }^{25}$ Inadequate aBMD gains in this study cannot be attributed to delayed puberty.

It is well known that oral corticosteroids impede bone formation. Only a small number of subjects received long term oral corticosteroids in this study, which may explain why we did not find an association with aBMD gain as reported previously. ${ }^{14}$ The negative association previously reported with inhaled corticosteroids in children/adolescents $^{17}$ was not observed in the longitudinal analysis, consistent with reports that inhaled corticosteroid usage in children and adolescents with asthma have a negligible effect on bone mass accrual. ${ }^{5051}$

In the absence of an easy to use and reliable objective method of measuring weight bearing activity, physical activity was assessed using a validated questionnaire. Questionnaires may not truly reflect actual physical activity due to altered perception. In adolescents the decline in the physical activity score during the study is consistent with earlier studies. ${ }^{52}$ Weight bearing exercise programmes have been associated with gains in bone mass in healthy children and adolescents over the short term, and this is an area for interventional studies in those with CF. ${ }^{53}{ }^{54}$ Dietary calcium intake in those with CF remained above the recommended level through out the study despite declining overall. Whether it is sufficient to compensate for impaired calcium absorption is unknown. ${ }^{55}$ Some caution is required when interpreting the results from the dietary diaries and physical activity questionnaires due to low participation rates, particularly in the adolescent groups.

25-hydroxyvitamin D is predominantly obtained by sunlight exposure with limited dietary absorption in healthy individuals. Vitamin D supplementation was not part of routine clinical care in the study population. The reason for the declining serum levels in children and adolescents with $\mathrm{CF}$ is unexplained and, in the absence of control subject longitudinal data, it is unclear whether this is unique to CF subjects. There was significant seasonal variation in those with $\mathrm{CF}$, although not the controls at study commencement (data not published), suggesting either a reduction in sunlight exposure during the winter months and/or altered vitamin D metabolism in those with CF. The skin cancer awareness campaign is very active in Queensland and whether the increasing use of more protective sunscreen is having an impact is unknown. Nevertheless, there were no associations between 25OHD and aBMD.

Histopathological evidence of the underlying disorder in young people with CF is limited. Elkin $^{56}$ reported low bone formation at a tissue and cellular level with no evidence of increased bone resorption in young adults with CF. These findings were not consistent with previously described metabolic bone disorders or the reports of increased urinary bone resorption markers. ${ }^{165657}$ The observations of inadequate aBMD accrual in this study are consistent with these findings. It has not been established whether the expression of dysfunctional CFTR affects osteoblast function. A moderately significant correlation between aBMD of CF patients and their mothers (obligate carrier of CFTR mutation) has been reported, ${ }^{11}$ indicating either that genetic predisposition determines $\sim 80 \%$ of peak bone mass and/or that dysfunctional CFTR influences bone mass accrual. ${ }^{58} 59$ Vitamin K insufficiency may contribute to low bone formation as vitamin $\mathrm{K}$ is required for $\gamma$-carboxylation of osteocalcin glutamic acid residues and uncarboxylated osteocalcin has a strongly reduced affinity for calcium. Higher uncarboxylated osteocalcin levels have been reported in those with CF. ${ }^{60-62}$

The primary concern of the low bone mass is the increased risk of fracture which has been documented in adolescents and adults with $\mathrm{CF}^{3}{ }^{8-10}$ In this study the incidence of limb fractures did not differ between the control and CF study groups. This may reflect the short study duration, the young population, inadequate study size, or selection bias on behalf of the control subjects.

Controversy exists around the best method of measuring and reporting bone mass measured by DXA in growing individuals. ${ }^{19} 2730$ We have elected to report aBMD, the measure from which Z-scores are commonly generated, rather than bone mineral content (BMC). When TB BMC was analysed (data not shown), similar results were found in keeping with others. ${ }^{63}$ One of the limitations of the study is that there were more missing data from the adolescents with CF which increases the likelihood of bias. As the adolescents who withdrew had greater disease severity, as indicated by $\mathrm{FEV}_{1}$ at study commencement, the differences in aBMD observed between CF and control groups reported here are likely to be underestimated.

In conclusion, suboptimal TB and FNt bone mass accrual in adolescents with CF, as measured by aBMD adjusted for age, 
sex and height Z-score, compared with local healthy controls was found in this study. This indicates that inadequate bone mass accrual during adolescence contributes to the low bone mass observed in adults with CF. A considerable proportion of the reduced gain in bone mass is explained by LTM and deteriorating disease status. At this point of our understanding, maximising nutritional status, encouraging and maintaining an active lifestyle, ensuring adequate 25OHD, and minimising the progression of chronic suppurative lung disease are required to optimise an individual's potential to acquire maximal peak bone mass and hence reduce the risk of future fracture.

\section{ACKNOWLEDGEMENTS}

The authors thank the patients and their families for their time in participating in this study, the staff involved with bone densitometry measurements for flexible scheduling, Catherine O'Neil for diligently processing the diet diaries, and Karen Cassidy for assisting in sample collection.

\section{Authors' affiliations}

H M Buntain, C E Wainwright, Department of Respiratory Medicine, Royal Children's Hospital, Herston, Brisbane, Australia

H M Buntain, R M Greer, Department of Paediatrics and Child Health, University of Queensland, Herston, Queensland, Australia

P J Schluter, Faculty of Health and Environmental Sciences, Auckland University of Technology, Auckland, New Zealand

S C Bell, Adult Cystic Fibrosis Unit and the Department of Medicine, University of Queensland, The Prince Charles Hospital, Chermside, Queensland, Australia

J C H Wong, Department of Nuclear Medicine and Bone Densitometry, Royal Brisbane and Women's Hospital, Herston, Queensland, Australia J Batch, Department of Endocrinology, Royal Children's Hospital, Herston, Brisbane, Australia

P Lewindon, Department of Gastroenterology and Hepatology, Royal Children's Hospital, Herston, Brisbane, Australia

The study was supported by Cystic Fibrosis Research Australia Pty Ltd, the Royal Children's Hospital Foundation - Working Wonders, and an Allen and Hanbury paediatric career development grant.

None of the authors has any competing interests with regard to this publication. Specifically, in relation to this study, none of the authors has in the last five years (1) accepted reimbursement or a fee that may in any way gain or lose financially from the results, (2) been employed by an organisation that may in any way gain or lose financially from the results, (3) hold any stocks or shares in an organisation that may in any way gain or lose financially from the results, (4) acted as an expert witness on the subject, or (5) any other competing financial interests.

\section{REFERENCES}

1 Bhudhikanok GS, Wang MC, Marcus R, et al. Bone acquisition and loss in children and adults with cystic fibrosis: a longitudinal study. J Pediatr 1998;133:18-27.

2 Conway SP, Morton AM, Oldroyd B, et al. Osteoporosis and osteopenia in adults and adolescents with cystic fibrosis: prevalence and associated factors. Thorax 2000;55:798-804.

3 Elkin SL, Fairney A, Burnett S, et al. Vertebral deformities and low bone mineral density in adults with cystic fibrosis: a cross-sectional study. Osteoporos Int 2001; 12:366-72.

4 Haworth CS, Selby PL, Horrocks AW, et al. A prospective study of change in bone mineral density over one year in adults with cystic fibrosis. Thorax 2002;57:719-23.

5 Haworth CS, Selby PL, Webb ME, et al. Low bone mineral density in adults with cystic fibrosis. Thorax 1999;54:961-7.

6 Kroger H, Huopio J, Honkanen R, et al. Prediction of fracture risk using axial bone mineral density in a perimenopausal population: a prospective study. $J$ Bone Miner Res 1995;10:302-6.

7 Schott AM, Cormier C, Hans D, et al. How hip and whole-body bone mineral density predict hip fracture in elderly women: the EPIDOS Prospective Study. Osteoporos Int 1998;8:247-54.

8 Henderson RC, Specter BB. Kyphosis and fractures in children and young adults with cystic fibrosis. J Pediatr 1994;125:208-12.

9 Aris RM, Renner JB, Winders AD, et al. Increased rate of fractures and severe kyphosis: sequelae of living into adulthood with cystic fibrosis. Ann Intern Med 1998; 128:186-93.

10 Rossini M, Del Marco A, Dal Santo F, et al. Prevalence and correlates of vertebral fractures in adults with cystic fibrosis. Bone 2004;35:771-6.
11 Ujhelyi R, Treszl A, Vasarhelyi B, et al. Bone mineral density and bone acquisition in children and young adults with cystic fibrosis: a follow-up study. J Pediatr Gastroenterol Nutr 2004;38:401-6.

12 Bailey DA, McKay HA, Mirwald RL, et al. A six-year longitudinal study of the relationship of physical activity to bone mineral accrual in growing children: the university of Saskatchewan bone mineral accrual study. J Bone Miner Res 1999; 14:1672-9.

13 Lu PW, Briody JN, Ogle GD, et al. Bone mineral density of total body, spine, and femoral neck in children and young adults: a cross-sectional and longitudinal study. J Bone Miner Res 1994;9:1451-8.

14 Bonjour JP, Theintz G, Buchs B, et al. Critical years and stages of puberty for spinal and femoral bone mass accumulation during adolescence. J Clin Endocrinol Metab 1991;73:555-63.

15 Gibbens DT, Gilsanz V, Boechat Ml, et al. Osteoporosis in cystic fibrosis. J Pediatr 1998; 113:295-300.

16 Baroncelli GI, De Luca F, Magazzu G, et al. Bone demineralization in cystic fibrosis: evidence of imbalance between bone formation and degradation. Pediatr Res 1997:41:397-403.

17 Buntain HM, Greer RM, Schluter PJ, et al. Bone mineral density in Australian children, adolescents and adults with cystic fibrosis: a controlled cross sectional study. Thorax 2004;59:149-55.

18 Sood M, Hambleton G, Super M, et al. Bone status in cystic fibrosis. Arch Dis Child 2001;84:516-20.

19 Hardin DS, Arumugam R, Seilheimer DK, et al. Normal bone mineral density in cystic fibrosis. Arch Dis Child 2001;84:363-8.

20 Haslam RH, Borovnicar DJ, Stroud DB, et al. Correlates of prepubertal bone mineral density in cystic fibrosis. Arch Dis Child 2001;85:166-71.

21 Henderson RC, Madsen CD. Bone mineral content and body composition in children and young adults with cystic fibrosis. Pediatr Pulmonol 1999;27:80-4

22 Laursen EM, Molgaard C, Michaelsen KF, et al. Bone mineral status in 134 patients with cystic fibrosis. Arch Dis Child 1999;81:235-40.

23 Mortensen LA, Chan GM, Alder SC, et al. Bone mineral status in prepubertal children with cystic fibrosis. J Pediatr 2000;136:648-52.

24 Salamoni F, Roulet M, Gudinchet F, et al. Bone mineral content in cystic fibrosis patients: correlation with fat-free mass. Arch Dis Child 1996;74:314-8.

25 Gronowitz E, Mellstrom D, Strandvik B. Normal annual increase of bone mineral density during two years in patients with cystic fibrosis. Pediatrics 2004; 114:435-42.

26 Carter DR, Bouxsein ML, Marcus R. New approaches for interpreting projected bone densitometry data. J Bone Miner Res 1992;7:137-45.

27 Lu PW, Cowell CT, SA LL-J, et al. Volumetric bone mineral density in normal subjects, aged 5-27 years. J Clin Endocrinol Metab 1996;81:1586-90.

28 Molgaard C, Thomsen BL, Prentice A, et al. Whole body bone mineral content in healthy children and adolescents. Arch Dis Child 1997;76:9-15.

29 Fewtrell MS. Bone densitometry in children assessed by dual $x$ ray absorptiometry: uses and piffalls. Arch Dis Child 2003;88:795-8.

30 Hogler W, Briody J, Woodhead HJ, et al. Importance of lean mass in the interpretation of total body densitometry in children and adolescents. J Pediatr 2003;143:81-8

31 Mosekilde L. Sex differences in age-related changes in vertebral body size, density and biomechanical competence in normal individuals. Bone 1990:11:67-73.

32 Mundy GR. Bone remodeling. In: Murray MF, eds. Primer on the metabolic bone diseases and disorders of mineral metabloism. 4th ed. Sydney: Lippincott William \& Wilkins, 1999:30-44.

33 Baron R. Anatomy and ultrastructure of bone. In: Murray MF, eds. Primer on the metabolic bone diseases and disorders of mineral metabolism. 4th ed. Sydney: Lippincott William \& Wilkins, 1999:3-11

34 Bonjour JP, Carrie AL, Ferrari S, et al. Calcium-enriched foods and bone mass growth in prepubertal girls: a randomized, double-blind, placebo-controlled trial. J Clin Invest 1997;99:1287-94.

35 Holick MF. Sunlight and vitamin D for bone health and prevention of autoimmune diseases, cancers, and cardiovascular disease. Am J Clin Nutr 2004;80(6 Suppl): 1678-88S.

36 Reiter EO, Stern RC, Root AW. The reproductive endocrine system in cystic fibrosis. I. Basal gonadotropin and sex steroid levels. Am J Dis Child $1981 ; 135: 422-6$

37 World Health Organization. Measuring change in nutritional status: guidelines for assessing the nutritional impact of supplementary feeding programmes for vulnerable groups. Geneva: World Health Organization, 1983.

38 Hibbert ME, Lannigan A, Landau LI, et al. Lung function values from a longitudinal study of healthy children and adolescents. Pediatr Pulmonol 1989;7:101-9.

39 Tanner JM. Growth at adolescence, 2nd ed. Oxford: Blackwell Scientific Publications, 1962

40 Duke PM, Litt IF, Gross RT. Adolescents' self-assessment of sexual maturation. Pediatrics 1980;66:918-20.

41 National Health and Medical Research Council. Recommended dietary intakes for use in Australia. Australia: National Health and Medical Research Council, 1998

42 Crocker PR, Bailey DA, Faulkner RA, et al. Measuring general levels of physical activity: preliminary evidence for the Physical Activity Questionnaire for Older Children. Med Sci Sports Exerc 1997;29:1344-9.

43 Adams N, Bestall JM, Jones PW. Fluticasone versus beclomethasone or budesonide for chronic asthma. Cochrane Database Syst Rev 2002; 1:CD002310.

44 Carrie Fassler AL, Bonjour JP. Osteoporosis as a pediatric problem. Pediatr Clin North Am 1995:42:811-24. 
45 Twisk JWR. Applied longitudinal data analysis for epidemiology: a practical guide. Cambridge: Cambridge University Press, 2003.

46 Diggle PJ, Heagerty P, Liang KY, et al. Analysis of longitudinal data, 2nd ed. Oxford: Oxford University Press, 2003.

47 Annual Report from the Australisian Cystic Fibrosis Data Registry. Cystic Fibrosis, Australia, 2001.

48 Haapasalo $\mathrm{H}$, Kontulainen S, Sievanen $\mathrm{H}$, et al. Exercise-induced bone gain is due to enlargement in bone size without a change in volumetric bone density: a peripheral quantitative computed tomography study of the upper arms of male tennis players. Bone 2000;27:351-7.

49 Young D, Hopper JL, Macinnis RJ, et al. Changes in body composition as determinants of longitudinal changes in bone mineral measures in 8 to 26 year-old female twins. Osteoporos Int 2001;12:506-15.

50 The Childhood Asthma Management Program Research Group. Long-term effects of budesonide or nedocromil in children with asthma. N Engl J Med 2000;343:1054-63.

51 Agertoft L, Pedersen S. Effect of long-term treatment with inhaled budesonide on adult height in children with asthma. N Engl J Med 2000;343:1064-9.

52 Britto MT, Garrett JM, Konrad TR, et al. Comparison of physical activity in adolescents with cystic fibrosis versus age-matched controls. Pediatr Pulmonol 2000;30:86-91.

53 luliano-Burns S, Saxon L, Naughton G, et al. Regional specificity of exercise and calcium during skeletal growth in girls: a randomized controlled trial. $J$ Bone Miner Res 2003;18:156-62.

54 McKay HA, Petit MA, Schutz RW, et al. Augmented trochanteric bone mineral density after modified physical education classes: a randomized school-based exercise intervention study in prepubescent and early pubescent children. J Pediatr 2000;136:156-62.

55 Aris RM, Lester GE, Dingman S, et al. Altered calcium homeostasis in adults with cystic fibrosis. Osteoporos Int 1999;10:102-8.

56 Elkin SL, Vedi S, Bord S, et al. Histomorphometric analysis of bone biopsies from the iliac crest of adults with cystic fibrosis. Am J Respir Crit Care Med 2002; 166:1470-4.

57 Greer RM, Buntain HM, Potter JM, et al. Abnormalities of the PTH-vitamin D axis and bone turnover markers in children, adolescents and adults with cystic fibrosis: comparison with healthy controls. Osteoporos Int 2003; 14:404-11.

58 Pocock NA, Eisman JA, Hopper JL, et al. Genetic determinants of bone mass in adults. A twin study. J Clin Invest 1987;80:706-10.

59 Gueguen R, Jouanny P, Guillemin F, et al. Segregation analysis and variance components analysis of bone mineral density in healthy families. J Bone Miner Res 1995; 10:2017-22.

60 Aris RM, Ontjes DA, Brown SA, et al. Carboxylated osteocalcin levels in cystic fibrosis. Am J Respir Crit Care Med 2003;168:1129.

61 van Hoorn JH, Hendriks JJ, Vermeer C, et al. Vitamin K supplementation in cystic fibrosis. Arch Dis Child 2003;88:974-5.

62 Conway SP, Wolfe SP, Brownlee KG, et al. Vitamin K status among children with cystic fibrosis and its relationship to bone mineral density and bone turnover. Pediatrics 2005; 115:1325-31.

63 Heaney RP. Bone mineral content, not bone mineral density, is the correct bone measure for growth studies. Am J Clin Nutr 2003;78:350-2.

\section{LUNG ALERT}

An important virus in asthma pathophysiology?

$\Delta$ Pifferi M, Maggi F, Andreoli E, et al. Associations between nasal torquetenovirus load and spirometric indices in children with asthma. J Infect Dis 2005;192:1141-8

T

here is evidence that viral respiratory infections may have an important role in asthma.

Torquetenovirus (TTV) is widespread in the general population, and increased loads

have been associated with decreased circulating CD3 and CD4 cells as well as increased circulating B cells and eosinophilic cationic protein (ECP), suggesting possible immunomodulatory effects. This group investigated, for the first time, the association between TTV carriage and spirometric indices in asthma.

The nasal secretions of 59 asthmatic children aged 7-16 years who had been stable for at least 2 months and 30 non-asthmatic non-atopic controls matched for age and residence were studied. 93\% of asthmatics and $83 \%$ of controls had TTV carriage (no significant difference). Because of the low number of subjects without TTV carriage, the analysis compared those with high TTV load and those with low or absent TTV load. No differences in forced expiratory volume in 1 second $\left(\mathrm{FEV}_{1}\right)$ or forced vital capacity (FVC) were found between cases and controls, but $\mathrm{FEV}_{1} / \mathrm{FVC}, \mathrm{FEF}_{25-75 \%}$, and $\mathrm{FEF}_{25-75 \%} / \mathrm{FVC}$ were all significantly lower in cases than in controls, and in cases with a high TTV load compared with those with a low TTV load. TTV load was also correlated with sputum ECP level.

The authors conclude that enhanced TTV replication may negatively affect the size and/or tone of small to medium airways in children with asthma, either directly or via the inflammatory response it elicits. Although they acknowledge that TTV replication may merely serve to identify children with abnormal airway or immune function, further work is warranted to prove that this virus is an important element in the pathophysiology of asthma.

D Waine 